\title{
Introduction to the Three-dimensional Scoliosis Treatment According to Schroth
}

\author{
Article Reprinted from
}

Physiotherapy, November 1992, vol 78, no 11 page 810-815.

This short article answers the question of "Scoliosis Straightening Exercises" better than any other that I know of in the English speaking world. The postural concepts in this article have striking parallels with the Pilates Exercise Methodology. It thus seems reasonable for health professionals to liaise with their local Pilates Studio for ongoing exercise based therapeutic programs - At least until such time as the Schroth method becomes known and accepted in the English speaking world.

Every effort has been made to accurately reproduce the original article, but no guarantees are made.

\section{Bruce Thomson}

Webmaster,

\section{http://www.easyvigour.net.nz}

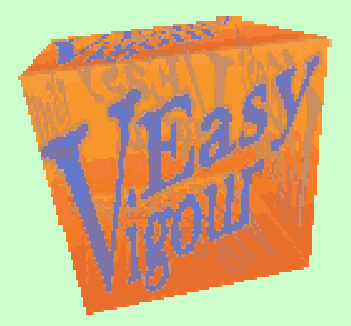




\section{Introduction to the Three-dimensional Scoliosis Treatment According to Schroth}

\section{Christa Lehnert-Schroth}

Physiotherapy, Nov 1992, vol 78, no 11

Key Words

Scoliosis, physiotherapy, exercise programme, Schroth.

\section{Summary}

The author gives an introduction to the basic principles of the three-dimensional scoliosis treatment according to Schroth, which is a physiotherapeutic approach to spinal deformity. The development of this specific treatment method by Katharina Schroth is described briefly. The paper gives a short review of the treatment methods as well as referring to aetiological and biomechanical aspects.

Introduction

To deal with her own scoliosis, my mother Katharina Schroth conceived a three-dimensional approach to treatment and designed an exercise programme to treat the various deformities and static changes of the scoliotic trunk additional to the curved spine. The method is taught to patients and physiotherapists at the Katharina Schroth Spinal Deformities Centre, Sobernheim, Germany. About 1,200 patients attend every year for an intensive course of in-patient physiotherapy lasting from four to six weeks depending on the referral from the consultan orthopaedic physician. They range in age from eight to 70 years, but children under ten years are treated only when accompanied by a person they know very well. The ratio of females to males is $7: 1$.

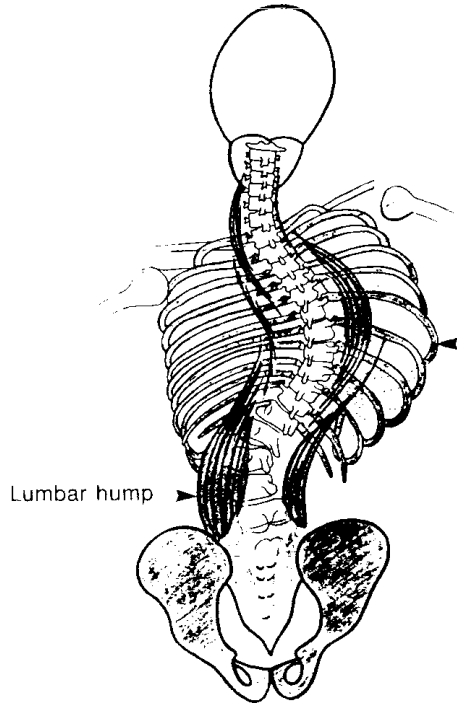

Fig 1: Scoliosis skeleton. The drawing shows the erector spinae muscle with varying thickness, shape and lateral asymmetry

\section{Scoliosis}

Definition and Description

Scoliosis (fig 1 ) is defined as a partly-fixed lateral curvature of the spine (Heine and Meister, 1972; Weinstein, 1989). Its origin may lie in paralysis, hereditary and genetic diseases, or other identifiable causes, but $80 \%$ of cases are idiopathic and, although researchers have investigated the aetiology (Moe and Byrd, 1987), no conclusive evidence as to cause has been documented. Of patients at the Katharina Schroth Spinal Deformities Rehabilitation Centre, $80 \%$ to $90 \%$ have idiopathic scoliosis. The deformity can, therefore, only be treated symptomatically.

A lateral curvature of the spine causes compensatory curves which may be much smaller but cannot be ignored in planning physiotherapy. A functional three-curve scoliosis can be observed in most scoliotic people, as illustrated in figure 2 . The ribs are rotated anteriorly on the concave side of the scoliotic curve and posteriorly on the convex side. Consequently, (a) there is a costal depression (rib valley) on the concave side and a thoracic gibbus (hump) on the convex side (fig 2b); there is a lumbar hump below the rib valley and a concavity below the thoracic hump (fig 2c); and (c) the shoulder girdle is drawn posteriorly above the rib valley and anteriorly above the costal convexity (fig $2 \mathrm{~d}$ ). Other symptoms include decreased spinal mobility on the convexity of the curve, back pain, psychological problems associated with the deformity, and cardio-respiratory dysfunction.

\section{Posture and Scoliosis}

Scoliosis must be seen as a multiplicity of postural disorders. The term 'posture' itself indicates more than a passive procedure or a permanent status but, to date, it has not been satisfactorily defined (Rizzi, 1979). Some authors define it as an act of balance (Basmajian, 1967; Tucker, 1969) without reference to an essential description. Taillard (1964) states that a good posture consumes minimal energy and does not stress musculature and connective tissue. A 'poor' posture may very easily change into a postural disorder, such as kyphosis, lordosis and scoliosis.

\section{Diagnosis}

If a patient bends forwards to touch his toes, slight dorsal asymmetries become evident (fig 3 ). The greater the rib prominence, the greater the torsion of corresponding vertebrae as ribs and musculature move with the rotation and develop dorsal elevations (humping of ribs, lumbar hump, 'elevated shoulder') or depressions (concavities of the back). X-ray assessment of the spinal curvature to determine the extent of the curve and to eliminate other possible causes (eg tumours) is generally carried out on the first visit. 

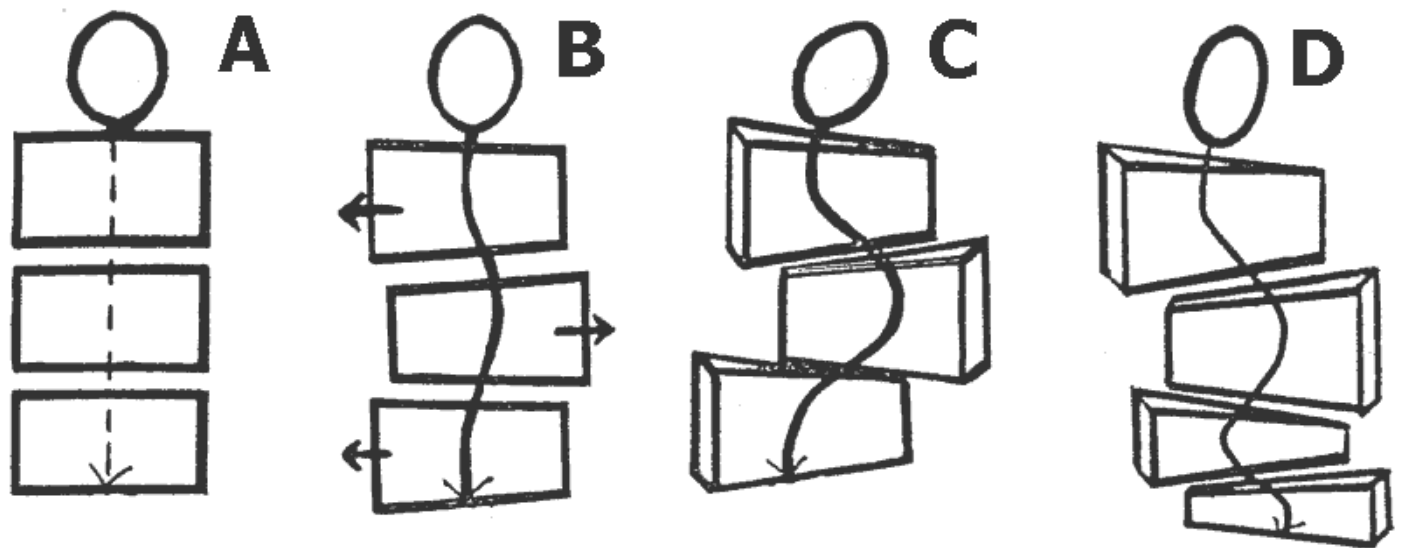

Fig 2: Block diagrams of trunk

(A) Subdivision into three rectangular superimposed blocks (pelvic girdle, rib cage, shoulder girdle)

(B) In scoliosis, three blocks of trunk deviate from vertical axis. This results in lateral shifting of spine

(C) The three blocks develop 'wedge-like' form, depending on severity of scoliosis, and rotate against each other around vertical axis. Ribs and spine follow these distortions. Scoliotic torsion is created

(D) Additional lumbosacral counter curvature. This pattern demands additional special pelvic corrections in order to influence existing pelvic torsion, explanations of which would go far beyond this paper.
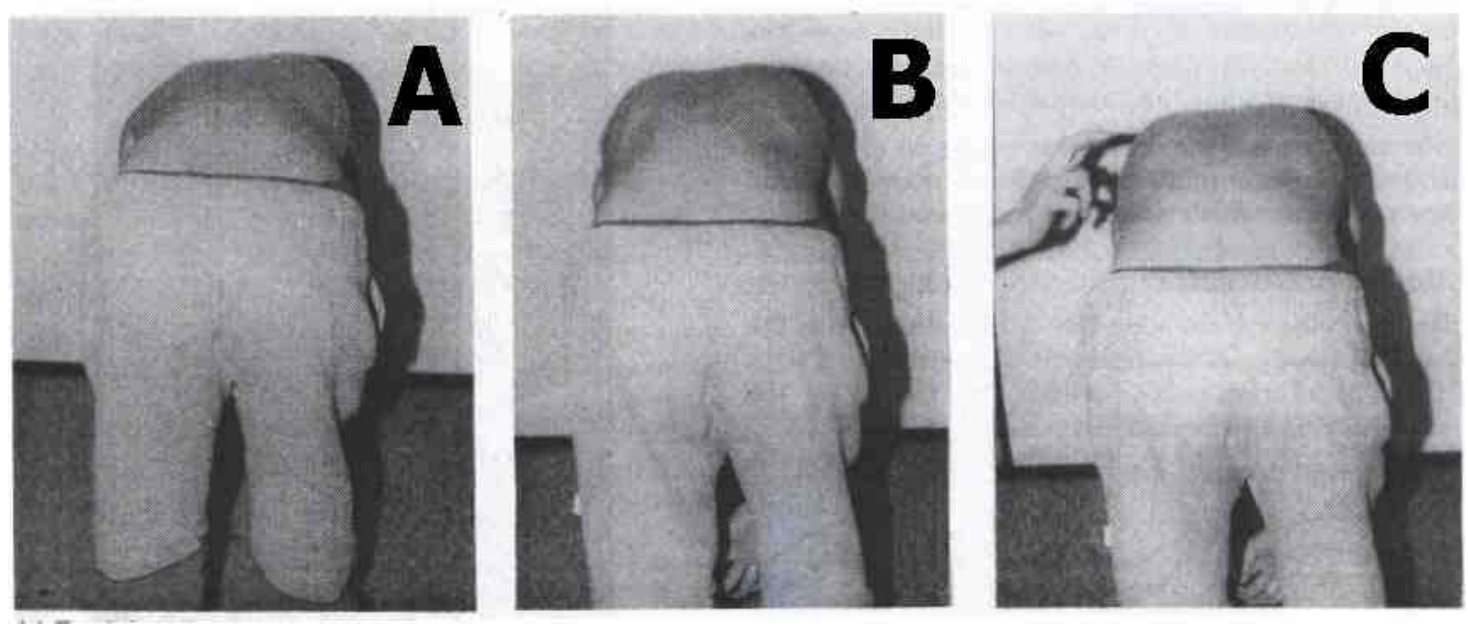

Fig 3: Eleven-year-old girl with idiopathic scoliosis

(A) Trunk bending forward; uni-lateral elevation of back on right side is clearly visible

(B) Patient practising rotational breathing according to Schroth with left ribs. Left concave side of back is filled, resulting in visible levelling

(C) Girl is stimulated by touches, thus provoking more effective exercise

\section{Prognosis}

The so-called thoracic 'hump' and the protrusion of a part of the hip result from progressive scoliosis -and increased rotation with lateral deviation of the trunk. In infantile idiopathic scoliosis this often leads to deformities of the rib cage with restriction of the respiratory, circulatory and cardiac function. The cardiorespiratory prognosis is not so bleak with adolescents and they do not usually suffer from major cardio-pulmonary restrictions in later life.

\section{Development of a Gibbus}

The presence of a hump may also lead to numerous psychological problems. Katharina Schroth is famous for her phrase: 'There is no hump, only torsioned ribs.' This is especially true for a scoliosis in the early stage of development. If nothing is done to counteract it, one lateral half of the back may quickly enlarge, because a gibbus develops as a consequence of imbalance of muscles and forces - mainly shifted ribs and associated muscles being pressed into the wrong direction, partly anteriorly and partly posteriorly and/or laterally. This 


\section{Scoliosis Straightening Exercises - Article Reprint courtesy of http://www.easyvigour.net.nz - 4 -}

The prognosis of the scoliosis itself depends upon various factors, especially the age of onset of a curvature angle and its magnitude. The greatest danger in idiopathic scoliosis occurs during the growing period of the adolescent years.

\section{Treatment}

Development of the Schroth Method

The treatment method was developed by Katharina Schroth in her youth. In the beginning, she intended only to correct her own appearance with her exercises and to normalise the scoliotic posture of her body. She used no talent but that of perception. She shaped her own body and then the bodies of her patients with her hands, guiding them with words. Doing this, she awoke in her patients a new awareness of body image for an imbalanced posture as well as for a balanced posture. By stimulation, she showed them where the narrowed rib segments were. She made her patients feel where to 'guide' their breath. She asked them to imagine or visualise forces and encouraged them to improve their performance gradually.

In a mirror, the patients could see how the scoliotic posture changed into a more favourable appearance and how the imbalance of skeleton and musculature was gradually transformed into an upright posture. By 'going over the middle' she initiated new movement patterns in her patients, as people with postural disorders perceive themselves as having an upright posture.

Step by step, Katharina Schroth formulated 'laws' according to which scoliosis increases and tried to apply therapeutic methods which would have the opposite effect. She said: 'We have to create the opposite appearance to what the scoliotic body shows.'

She used ribs as long lever arms acting on a torsioned thorax by anterior rotation. Thus she discovered and used the technique which she called 'rotational breathing'.

\section{Rotational Breathing}

Because ribs are connected by articulations with the lateral processes of the vertebrae (fig 4), they can, with the help of respiration, reduce the torsion of the trunk during the Schroth exercises.

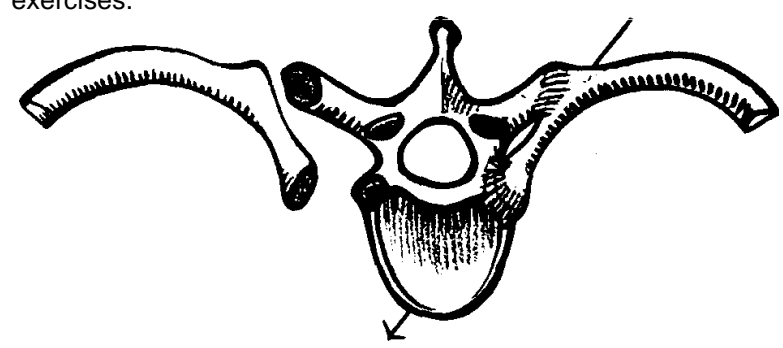

Fig 4: Thoracic vertebra. Left: Rib is dislocated from vertebra. Right: Rib heads and tubercules are connected by articulations with vertebra. By the axis which has been created in this way (see arrow), raising and lowering of ribs during respiration is made possible (after Mollier, 1938)

It is unwise to depress the prominent trunk sections before there is enough space to accommodate them. On the depressed (concave) side the ribs which have sunk inwards and downwards need to be widened from the inside by specific respiratory exercises. Following the idea of doing exactly the opposite of what the body shape presents, they have to be lifted to the outside (laterally) and upwards (cephally). results automatically in better ventilation of the pulmonary half on the side of the rib hump, whereas the other side - the concave (depressed) side - is less well ventilated. The shifting of the centre of gravity leads to a static imbalance.

By doing so, a wider space is created which allows the ribs to be moved backwards. This is effected by a 'respiratory thoracic movement' along the sides of an imaginary right-angle: laterally + cephally + posteriorly $=$ three-dimensionally. The counterpart of the posterior rib hump is the narrowed anterior thorax, as it is the same ribs which are rotated posteriorly that cause the anterior compression.

In the Schroth treatment, these ribs are moved anteriorly and upwards and rotated forwards and inwards or threedimensionally with the help of respiration ('rotational breathing'). At the same time the subject is always urged to think about lowering the diaphragm.

\section{Scoliotic Statics due to Postural Disorder}

Katharina Schroth could see the scoliotic statics of the body especially in cases of major scolioses - in nearly every case a postural disorder is present in the sagittal (anterior-posterior) plane with an anterior protrusion of the pelvis. Thus, the trunk deviates posteriorly from the lumbar region. The head swings again over the centre of gravity. This is due to the body equilibrium, which develops into an imbalanced state and finally adapts to the acquired scoliotic static. This is true for scoliotic patients when seen from the rib hump side (fig 5).

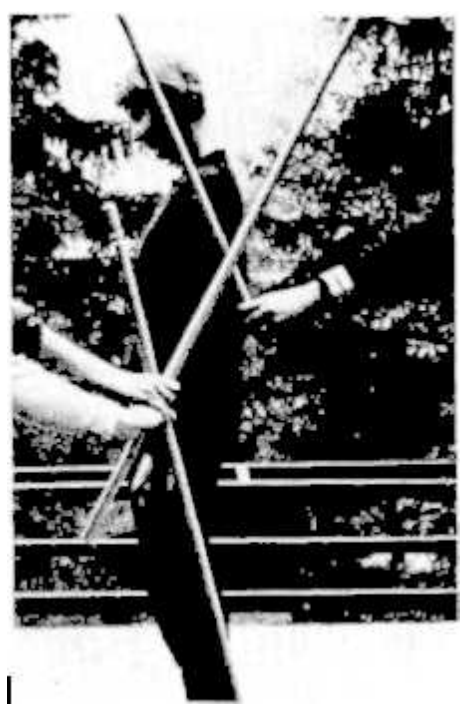

Fig 5: 19-year-old girl convinced she has an upright posture. Bars however prove scoliotic posture. Beginning from the feet, pelvis is carried anteriorly. For reason of balance, body leans posteriorly beginning from lumbar region. The head swings again anteriorly over the centre of gravity. Body forms 'double broken line'

For this reason, postural disorder is first corrected by skeletal correction: pelvis backwards, trunk forwards, 'creating the opposite shape' (fig 6). The same principle is followed when correcting the scoliotic static in the frontal plane with the lateral displacement of the individual body sections. The pelvis which is unilaterally protruding is taken in towards the line of gravity (figs $7,8)$. This results automatically in making the trunk more erect. Only this change in posture makes it possible to use rotational breathing effectively. 

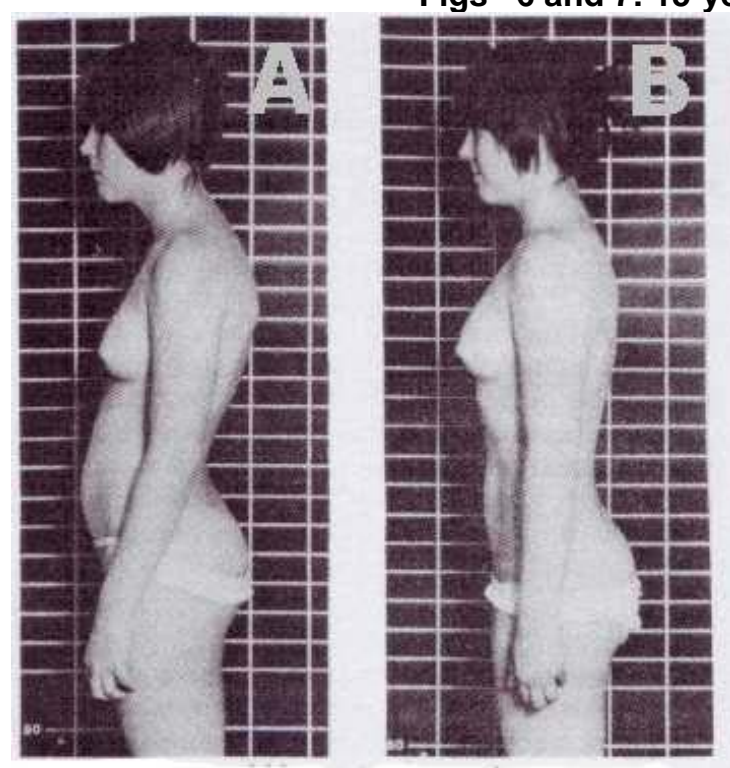

(A) Initial state as girl in fig 5, with postural disorder in sagittal plane

(B) After four weeks intensive treatment according to Schroth, showing postural correction

When trunk and spine have reached their optimal length, the trunk sections which are rotated against each other are able to move without mutual interference.

Often children and adolescents bring along their braces which have been adapted at home and which should be worn while they are not exercising. There are some exceptions which make it necessary to exercise in a brace, such as when it has to be worn 23 hours a day. Nowadays several good braces exist, such as the Cheneau and

Boston braces, which work in conjunction with the Schroth programme and do not inconvenience the patient. They are of tremendous benefit and help in supporting the obtained corrections.

\section{Working Principles According to Schroth}

After Katharina Schroth had worked with patients by shaping and forming their bodies, she discovered the 'laws' according to which she was working. She acquired a great deal of practical experience before she wrote about her theory. Gradually, she improved her theoretical knowledge so that it could be taught to others.

Katharina Schroth divided the trunk into three blocks which can be shifted against each other (fig 8). She recognised that the pelvic and the shoulder girdles are rotated into the same direction and that the middle block, the rib cage, is oriented in the opposite direction - in the sagittal plane as well as in the frontal plane. The more these blocks shift against each other, ie the more they deviate from a vertical line, the more they rotate also in the transverse plane (about the vertical body axis). The body becomes less and less upright and 'crumbles', because all parts of the body which deviate from the vertical line are drawn downwards by gravity. For this reason, active extension is a prerequisite of successful exercising.

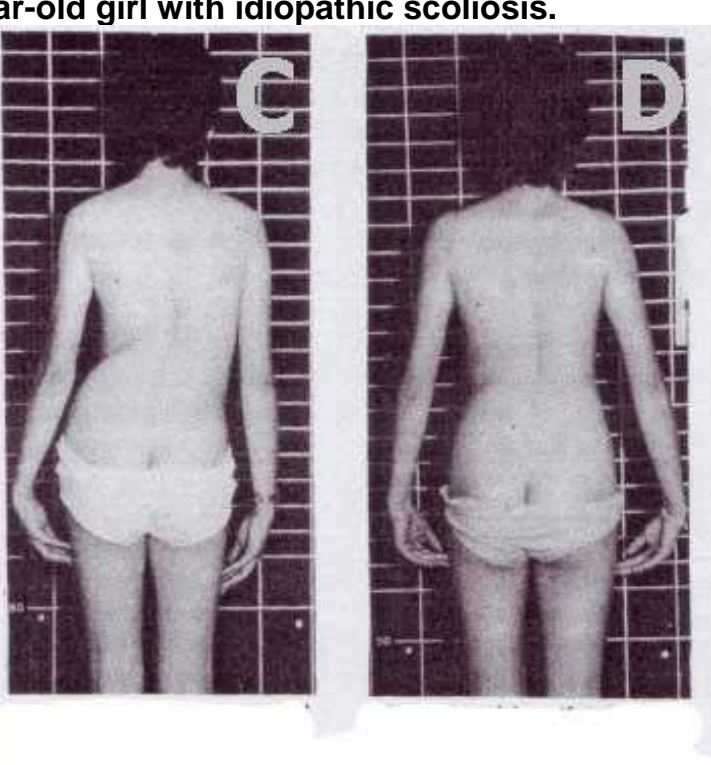

(C) Shifting and torsion of three blocks of trunk against each other, changing body statics.

(D) Same girl at the end of six-week therapy. Three blocks of trunk have largely been realigned vertically, eliminating scoliotic body statics and improving personal appearance

Fig 8: Diagram of girl in fig 7. Overstretched muscles are shortened, contracted muscles extended. Scoliotic body statics are largely corrected (from LehnertSchroth, 1991)

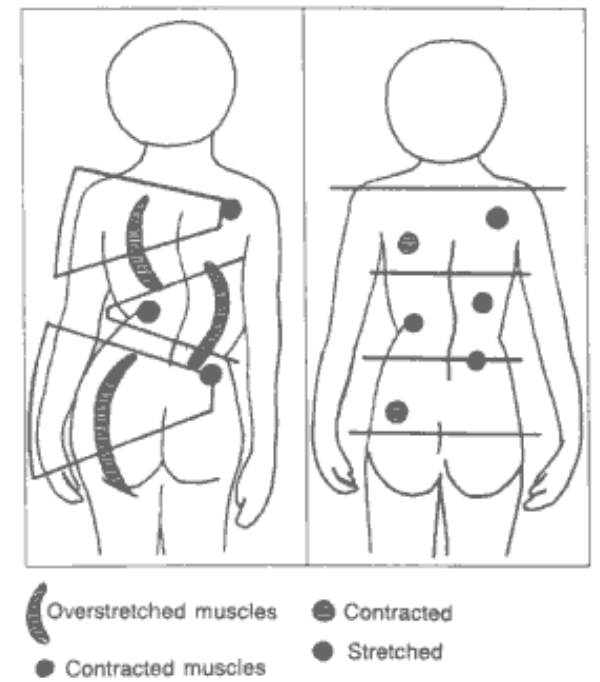


The elongation is connected to active curve correction and realignment of the trunk segments which have. deviated laterally. This is completed by active derotation of the three trunk segments rotated against each other. This is achieved by appropriate starting positions for orthopedic exercises as well as by rotational breathing.

It has been shown that this rotational breathing leads to a significant increase in rib mobility and thus vital capacity (Weiss, 1991a), and in sagittal respiratory excursion (Weiss, 1991b) which is of great importance in improving a flat back. Each exercise is connected with the feeling of the new movement pattern which aims at achieving normality. This is a facilitation process, ie the starting of a mental re-education by consciously repeated derotative exercises which are finally recognized by the patient's subconscious mind and then carried out automatically. The subject establishes a balance between what he sees in the mirror and what he feels. The continuous muscular training results in a re-education of the scoliotic posture into a corrected upright posture, so that new movement patterns are mentally accepted and the patient is capable of adopting a corrective posture at any time on her own.

The new postural pattern is the basis for learning a new movement pattern, i.e. patients also need to integrate acquired body and corrective perception into activities of daily living. So that the good results from exercise can later develop into a good body shape, active stabilization is necessary. According to Schroth, this is performed during the expiration phase in the form of isometric tension exercises of the muscle layer so that changed muscle tension in the correction becomes perceptible to the patient. Previously inactive muscles are reactivated. On the 'thick' side, the muscles are overstretched (overstrained) and weak; on the narrowed side, muscles are contracted. They have lost their natural muscle tone and are no longer ready to work. This is counteracted by stretching the shortened muscles with appropriate exercises in suitable starting positions and when they start to work again in a tensioned state. This automatically gives the elongated muscles a contractive stimulus.

These points are essentially connected. Moreover, each exercise must begin with the feeling of the correct movement and the conscious capability of transferring this movement into a corrected body posture. The new posture finally becomes established and leads to a confirmation of the patient's new selfimage.

\section{Outcomes}

\section{Cosmetic Improvement}

An additional advantage of this treatment is improvement in appearance. Patients are photographed, naked, from all four sides at the beginning and end of their six-week therapy. By means of these photographs it is possible to explain scoliotic statics to them. The photographs are an important tool during the exercises. They encourage subjects to exercise alone at home, especially if step-by-step success is apparent. For the patients, the degrees of angle of the spine which can be measured with the help of the roentgenogram are largely of' secondary importance. Cosmetic results

matter more to them; they want to see that their 'hump back' becomes smaller, because that is what troubles them most.

\section{Motivation to Exercise Alone}

The explanations which are always given to patients develop their self-confidence and convince them of the usefulness of the procedure, which also enhances their motivation to do the exercises. Each scoliotic person has to exercise throughout his life. During the first period of therapy, they exercise five to six hours daily. At home, they can reduce intensive daily exercise initially to 90 minutes or an hour. We have met patients again decades later and upon questioning they answered that they still exercise according to the Schroth method for ten minutes a day. That is motivation! In any case, the patients must comply with the treatment because this is essential for its success.

\section{Reduction of Pain}

Older patients in particular suffer from pain as a result of the scoliotic posture. The development of spinal curves leads to a uni-lateral tensioning or contusion of the nerves as well as to multiple joint dysfunction. This creates pain. According to our records, this pain improves or disappears in $85 \%$ of the patients, sometimes after only a few weeks of therapy, which thus helps to improve the quality of life.

\section{Improvement in Pulmonary Function}

Vital capacity increases measureably in about $95 \%$ of the patients (Lehnert-Schroth, 1991): 22\% obtained an improvement of up to $600 \mathrm{ml}$ vital capacity after six weeks and $11 \%$ obtained an improvement of up to $800 \mathrm{ml}$ in the same period. There were also patients who showed an increase of more than $1,000 \mathrm{ml}$ vital capacity within the six-week therapy. This represents an enormous improvement in health which also benefits from exercising in the open air if weather permits.

\section{Cardiac-circulatory Training}

Cardiac and circulatory functions are improved not only by a specific respiratory therapy but also by intensive muscle training. In bad weather, exercises are conducted in large halls equipped with suitable appliances for specific group exercises, which are complemented by individual exercises.

\section{Conclusion}

The Schroth technique is a scoliosis-specific back school. In the treatment programme, all possibilities for postural correction, including respiration, are used in order to enable the patients to help themselves. The patients learn to acquire a certain feeling which helps them to see and understand the different stages they must go through until they reach the best possible correction. In this way, they learn to accept that treatment will be long-term. The procedure also enables them to avoid behaviour during everyday activities which could increase progression of their scoliosis. Only the maintenance of postural correction during everyday activities can prevent progression in the long run. Courses for physiotherapists are held in Sobernheim so that scoliosis patients can be treated according to the Schroth method in their home towns or close to them. 
In some cases, where patients have already undergone surgery, it is possible to improve pulmonary function and maintain surgical results. Although surgery fuses the spine there is some loss of curve correction over time. To date most surgical techniques do not reduce the rib hump and thus specific physiotherapeutic exercises are aimed at maintaining surgical results. After a therapeutic course at the Katharina Schroth Spinal Deformities Centre, patients revisit the orthopaedic surgeons in their home towns, continue therapy and thus increase stabilisation of the acquired new postural feeling.

The three-dimensional scoliosis treatment according to Schroth has been taught since 1958 at the School of Kinesitherapy in Brussels. It is also taught in Spain, Austria, France, Switzerland, Germany and Brazil.

\section{Author}

Christa Lehnert-Schroth PT is head of the Katharina Schroth Spinal Deformities Rehabilitation Centre, Sobernheim, Germany.

\section{Address for Correspondence}

Mrs C Lehnert-Schroth PT, Katharina Schroth Spinal Deformities Rehabilitation Centre, DGKS (Geschaftsstelle), Leinenbornerweg 44, 6553 Sobernheim, Germany.

\section{References}

1) Basmajian, J (1967). Muscles Alive, Williams and Wilkins, Baltimore.

2) Heine, J and Meister, R (1972). `Quantitative Untersuchungen der Lungenfunktion und der arteriellen Blutgase bei jugendlichen. Skiliotikern mit Hilfe eines funktionsdiagnostischen Minimalprogrammes', Zeitschrift fuer Orthopaedie und Ihre Grenzgebrite. 110, 56-62.

3) Lehnert-Schroth, C (1991). Dreidimensionale Skoliosebehandlung (4th edn) Gustav Fischer Verlag, Stuttgart.
4) Lehnert-Schroth, C (1987). `Die dreidimensionale Skoliosebehandlung nach Schroth. Eine konservative Behandlungsweise der Ruckgratverkrummung', Deutsche Krankengymnastische Zeitschrift, 11, 751756.

5) Moe, J H and Byrd, J A (1987). 'Idiopathic scoliosis' in: Bradford. D S, Lonstein, J E and Moe, J H et al (eds) Moe's Textbook of Scoliosis and other Spinal Deformities, WB Saunders, Philadelphia, pages 191232.

6) Mollier, S (1938). Plastische Anatomie, Verlag J F Bergmam,i. Munich.

7) Rizzi, A M (1979). Die menschliche Haltung und die Wirbelsaule. Hippokrates, Stuttgart.

8) Taillard, W (1964). 'Die Klinik der Haltungsanomalien' in: Belart;. S(ed) Funktionsstorungen der Wirbelsaule, Huber Company Bern.

9) Tucker, W E (1969). Home Treatment and Posture, E \& S Livingstone, Edinburgh.

10) Weinstein, $S L$ (1989). 'Die idiopathische Adoleszentenskoliose Haufigkeit und Progression unbehandelter Skoliosen'. De: Orthopade, 18, 74-86.

11) Weiss, H R (1991a). 'The effect of an exercise programme on vital capacity and rib mobility in patients with idiopathic scoliosis', Spine, 16, 1, 88-93.

12) Weiss, H R (1991b). 'Aspects biomechaniques d'exercices specifiques clans le traitement de la scoliose', Proceedings of the 19th annual meeting of GEKTS, Modena, October 18-19.

Physiotherapy, November 1992, vol 78, no 11 